\title{
A reduced model of cavitation physics for use in sonochemistry
}

\author{
By BRian D. STOREY AND ANDREW J. SzERI \\ Department of Mechanical Engineering, University of California, \\ Berkeley, CA 94720-1740, USA
}

Received 21 February 2000; accepted 12 January 2001

Sonochemistry involves focusing acoustic energy through cavitation bubbles to increase chemical activity. The violent bubble collapses lead to temperatures of several thousand kelvin, which drive chemical reactions. In previous work, we gave a detailed computational model of a single bubble collapse, taking into account phase change, mass diffusion, heat diffusion and chemical reactions. All of these phenomena are important in determining the conditions at collapse. The present work involves development of a much simpler model that includes all the physics relevant to the determination of the reaction products. Comparisons with the more detailed computations are made; the reduced model is found to provide reasonable results. Furthermore, it is shown that many of the observed trends in sonochemistry are reflected in the trends observed in the behaviour of a single 'representative' bubble.

Keywords: sonoluminescence; sonochemistry; bubble dynamics

\section{Introduction}

Sonochemistry involves enhancing or promoting chemical reactions with ultrasonic acoustic energy. The diffuse acoustic energy is focused through cavitation bubbles, which grow and collapse in response to the oscillatory pressure. Violent collapses, due to the nonlinearity of the bubble dynamics, can produce temperatures of several thousand kelvin and pressures of several thousand atmospheres. These extreme conditions can lead to chemical reactions in the interior of the bubble; the products of the reactions are dispersed into the liquid. Some applications and techniques were recently reviewed by Suslick et al. (1999), Mason (1999), Thompson \& Doraiswamy (1999), and von Sonntag et al. (1999).

Several models of the dynamics of single bubbles have been proposed. The simplest equation is the Rayleigh-Plesset equation (RPE): a nonlinear ordinary differential equation (ODE) describing the radial dynamics (see Leighton 1994; Young 1989). This equation is derived for slow bubble motions; many of the assumptions on which it is based are invalid at the time of collapse.

More recently, the gas dynamics inside strongly collapsing bubbles has been studied through compressible Navier-Stokes simulations (Vuong \& Szeri 1996; Storey \& Szeri 1999, 2000; Moss et al. 1999). These detailed models have been used to show that the state of the gas on collapse is strongly influenced by such processes as heat transfer, mass transfer, chemical reactions and non-uniform pressure in the bubble interior. 
These physical phenomena have been left out of the traditional Rayleigh-Plesset formulation.

Others have presented modifications to the RPE taking into account a variety of phenomena in a variety of approximations. For example, heat-transfer effects were taken into account by Prosperetti et al. (1988), Plesset \& Hsieh (1960) and Plesset \& Zwick (1954). Compressibility of the liquid was taken into account by Prosperetti \& Lezzi (1986). Non-equilibrium phase change was included by Fujikawa \& Akamatsu (1980) and Yasui (1997a). Diffusion of vapour was studied by Nigmatulin et al. (1981). Chemical reactions have been included by Yasui (1997b), Sochard et al. (1997), Gong \& Hart (1998), Colussi et al. (1998) and Kamath et al. (1993). Nonequilibrium phase change and diffusion of vapour were considered very recently by Colussi \& Hoffmann (1999).

A problem with the simplified Rayleigh-Plesset models is that many of the assumptions are violated during the violent collapse. It is not known how well the approximations predict the actual conditions in the bubble interior. On the other hand, a weakness of the detailed models is that their complexity does not allow for extensive parameter studies.

In the present work, we develop a reduced model that does take into account phase change, mass diffusion, heat diffusion, non-uniform pressure and chemical reactions in a simple way. The results are compared with the more detailed Navier-Stokes calculations presented in our earlier work. Good agreement is shown between the two approaches when one considers such issues as peak temperatures and quantity of radical species produced. More importantly, the reduced model can predict the same trends as the more detailed calculations. Finally, we shall demonstrate that the simplified single-bubble model is able to predict a variety of trends observed in sonochemistry experiments.

\section{Formulation}

This formulation is based on the physical observations made from our computational studies of an argon bubble in water around standard atmospheric conditions (Storey \& Szeri (2000), hereinafter referred to as S\&S). In S\&S we solved the full Navier-Stokes equations inside the bubble for a diffusive, reactive, multi-species gas (including the water vapour). In what follows we shall simplify the model considerably, while retaining all the essential physics relevant to the determination of reaction products.

\section{(a) Radial dynamics}

The starting point is the RPE, the derivation of which may be found in many other references (see, for example, Leighton 1994). It is useful to make this equation dimensionless using the length-scale of the ambient bubble radius, $R_{0}$, the timescale of the frequency of acoustic forcing, $1 / \omega$, and the pressure scale of the ambient pressure, $P_{0}$. In dimensionless variables this equation becomes

$$
\ddot{R} R+\frac{3}{2} \dot{R}^{2}=E u\left(P_{1}-1+P_{\mathrm{A}} \sin (2 \pi t)+M a_{1} R \dot{P}_{1}\right),
$$

where $R$ is the bubble radius, the dot denotes a time derivative, $P_{1}$ is the pressure in the liquid at the bubble wall, and $P_{\mathrm{A}}$ is the amplitude of the acoustic forcing. The 
time derivative of the liquid pressure term is the traditional liquid compressibility correction (Prosperetti \& Lezzi 1986). The dimensionless pressure in the liquid is

$$
P_{\mathrm{l}}=P_{\mathrm{g}}+M a_{\mathrm{g}} R \dot{P}_{\mathrm{g}}-\frac{1}{E u}\left(\frac{1}{W e R}+\frac{\dot{R}}{R e_{1} R}\right),
$$

where $P_{\mathrm{g}}$ is the pressure in the gas (including vapour). The time derivative of the gas pressure term is an approximation for the non-uniform pressure in the interior of the bubble (Moss et al. 2000). There are five dimensionless parameters: Euler number $E u=P_{0} / \rho_{1} R_{0}^{2} \omega^{2}$; Weber number $W e=\rho_{1} R_{0}^{3} \omega^{2} / 2 \sigma$; liquid Mach number $M a_{1}=R_{0} \omega / c_{1}$; gas Mach number $M a_{\mathrm{g}}=R_{0} \omega / 3 c_{\mathrm{g}}$; and liquid Reynolds number $R e_{1}=\omega R_{0}^{2} / 4 \nu_{1}$. Here, $\rho_{1}$ is the liquid density, $\sigma$ is the surface tension, $c_{1}$ is the speed of sound in the liquid, $c_{\mathrm{g}}$ is the temperature-dependent speed of sound in the gas, and $\nu_{1}$ is the viscosity of the liquid.

As far as the radial dynamics is concerned, the two most important parameters are $E u$ and $P_{\mathrm{A}}$. We becomes important only for bubbles small enough that their surface tension causes significantly larger pressures within the bubble than in the liquid outside. The other parameters $\left(M a_{1}, M a_{\mathrm{g}}, R e_{1}\right)$ are associated with damping and have minor influence on a single bubble collapse. These dimensionless parameters (or other dimensionless groupings) should be kept in mind when one is concerned with varying input parameters. For example, single-bubble results are often presented with variations in both ambient radius and driving frequency. Under circumstances where $W e$ is not important, variations in $R_{0}$ and $\omega$ may really be the same through the Euler number.

\section{(b) Non-equilibrium phase change}

From the detailed calculations we found non-equilibrium phase change to be of paramount importance. Therefore, we use an evolution equation for the number of vapour molecules contained in the interior, rather than assume a constant vapour pressure. Non-equilibrium phase change does not significantly influence the bubble dynamics, but is very important for predicting the quantity of vapour at the collapse. The equation for number of moles of vapour, $n_{\mathrm{v}}$, in dimensionless variables is (Carey 1992)

$$
\dot{n}_{\mathrm{v}}=-\tau_{\mathrm{c}} \frac{n_{\mathrm{v}}-P_{\mathrm{sat}}\left(T_{0}\right) R^{3}}{R},
$$

where $P_{\text {sat }}\left(T_{0}\right)$ is the dimensionless saturation pressure at the ambient liquid temperature, $T_{0}$, and $\tau_{\mathrm{c}}=\left(3 \hat{\sigma} / R_{0} \omega\right)\left(\bar{R} T_{0} / 2 \pi M_{\mathrm{v}}\right)^{1 / 2}$ is the evaporation time constant. Here, $\hat{\sigma}$ is the accommodation coefficient, $\bar{R}$ is the universal gas constant, and $M_{\mathrm{v}}$ is the molecular weight of the vapour. The equation is made dimensionless by the initial number of moles $n_{0}=P_{0} 4 \pi R_{0}^{3} / 3 \bar{R} T_{0}$.

In (2.3) there are two new parameters, $\tau_{\mathrm{c}}$ and $P_{\text {sat }}$. Neither has much impact on the radial dynamics, but both are crucial in determining the quantity of vapour contained in the bubble, and hence the quantity of radical species produced.

\section{(c) Heat transfer}

Heat transfer is taken into account in a simple and approximate way. Heat-transfer effects are controlled by two competing time-scales. The dimensionless time-scale for 
heat to diffuse from the centre to the wall is

$$
\tau_{\text {diff }, \mathrm{h}}=\frac{R e_{\mathrm{g}} P r}{4 R},
$$

where $R e_{\mathrm{g}}=R_{0}^{2} \omega / \nu_{\mathrm{g}}$ is the Reynolds number of the gas, $\operatorname{Pr}=\nu_{\mathrm{g}} / \alpha_{\mathrm{g}}$ is the Prandtl number, $\nu_{\mathrm{g}}$ is the viscosity of the gas, and $\alpha_{\mathrm{g}}$ is the thermal diffusivity of the gas. The representative time-scale of the radial dynamics is given as

$$
\tau_{\text {dyn }}=R /|\dot{R}| \text {. }
$$

When the dynamic time-scale is longer than the heat-transfer time-scale, then the bubble motions are slow enough that the bubble interior remains at the ambient liquid temperature, i.e. the bubble behaves isothermally. When the heat-transfer time-scale is longer than the dynamic time-scale, then the bubble motions are so rapid that heat has insufficient time to escape from the bubble interior and the interior behaves adiabatically. A major simplification we employ to develop a model of reduced complexity is to treat the gas as either isothermal or adiabatic. Similar approaches to the heat-transfer problem have been taken before (see, for example, Noltingk \& Neppiras 1950; Neppiras \& Noltingk 1951). The adiabatic/isothermal behaviour determines how to compute the pressure in the gas mixture, to which we turn our attention in $\S 2 f$.

\section{(d) Mass transfer}

Mass diffusion of vapour inside the bubble is taken into account in the same way as the heat transfer. The mass-diffusion time-scale is

$$
\tau_{\text {diff }, \mathrm{m}}=\frac{R e_{\mathrm{g}} S c}{4 R}
$$

where $S c=\nu_{\mathrm{g}} / D_{\mathrm{v}}$ is the Schmidt number, and $D_{\mathrm{v}}$ is the mass diffusivity of the vapour.

When the dynamic time-scale is longer than the mass-diffusion time-scale, the interior is of uniform composition and the number of moles of vapour evolves according to (2.3) (analogous to the isothermal behaviour). When the mass-diffusion time-scale is longer than the dynamic time-scale, the vapour has insufficient time to diffuse from the interior to the wall. In this case, the amount of vapour contained in the bubble remains constant (analogous to the adiabatic behaviour). As with heat transfer, we switch between these two extreme behaviours in order to simplify mass transfer in the model. Hence, we set the right-hand side of (2.3) to zero when $\tau_{\text {dyn }}<\tau_{\text {diff,m }}$. Details on the role of transport of water vapour in the interior of bubbles are discussed extensively in S\&S.

\section{(e) Chemical kinetics}

The chemical reactions are taken into account with finite-rate chemical kinetics; chemical kinetics is reviewed in several references (see, for example, Gardiner 1984). The reaction mechanism consists of several forward and reverse elementary reactions; the sum of these elementary reactions gives the total change in an individual species. The evolution equation for the moles of each species is

$$
\dot{n}_{i}=\Gamma_{i} R^{3},
$$

Proc. R. Soc. Lond. A (2001) 
where $\Gamma_{i}$ is total change in concentration of species $i$ (which comes from the reaction equations (not shown)). In this paper we only consider $\mathrm{H}_{2}-\mathrm{O}_{2}$ chemistry; the details of the mechanism we used are given by Maas \& Warnatz (1988). One could easily use a chemical kinetics software package, such as CHEMKIN (Kee et al. 1994), to compute $\Gamma_{i}$ and extend this model to more complex chemical systems.

\section{(f) Pressure in the gas-vapour mixture}

The equation to determine the pressure depends on the process given by the heattransfer condition discussed in $\S 2 c$. When the bubble contents evolve isothermally, we assume that no reactions are occurring. The dimensionless pressure is given by the ideal gas law as

$$
P_{\mathrm{g}}=\frac{n_{\mathrm{v}}+n_{\mathrm{nc}}}{R^{3}}
$$

where $n_{\mathrm{nc}}$ is the number of moles of the non-condensible gas, which is regarded as a constant. In other words, we neglect rectified diffusion. Of course, rectified diffusion is an important ingredient in understanding the long-time evolution of bubble populations, or, indeed, in understanding how the present state of a bubble cloud came into being. In the present work, the focus instead is on chemical production per acoustic cycle, without consideration of these longer term effects.

When the bubble contents evolve adiabatically, the temperature rises and chemical reactions occur. During the adiabatic process the pressure is given by

$$
\dot{P}_{\mathrm{g}}=(1-\gamma) \sum_{i}\left(H_{i}-\bar{C}_{p} T\right) \Gamma_{i}-3 \gamma P_{\mathrm{g}} \frac{\dot{R}}{R},
$$

where $\gamma$ is the ratio of specific heats, $H_{i}$ is the partial enthalpy of species $i$, and $\bar{C}_{p}$ is the average molar specific heat of the gas mixture (see Gardiner 1984). Equation (2.6) is derived in a straightforward manner: one applies the first law of thermodynamics to an adiabatic, constant-mass bubble consisting of a mixture of chemically reacting ideal gases. We assume that all quantities are spatially uniform in the interior for the derivation of (2.6). The first term on the right-hand side is a consequence of the chemical reactions; without it (2.6) could be solved to yield the usual polytropic expression for an adiabatic process. We note that the kinetic energy of the gas was neglected when deriving this pressure equation. This term was checked and always found to be small.

\section{(g) Summary}

We assume that in sonochemistry applications, the cavitation is transient and the bubble breaks apart at the collapse, dispersing the chemical contents into the liquid (S\&S; Colussi et al. 1998). Therefore, we are only interested in the first expansion and violent collapse, a typical example is shown in figure 1.

At the beginning of the acoustic cycle, the bubble expands slowly to a maximum radius. The time-scale arguments of $\S \S 2 c$ and $2 d$ characterize this expansion as isothermal and the composition of the bubble interior is uniform. Therefore, at this time we solve (2.1) and (2.2) for the radius, (2.3) for the number of moles of vapour, and (2.5) for the pressure. No reactions occur during this part of the cycle and the right-hand side of equations (2.4) are set to zero. 


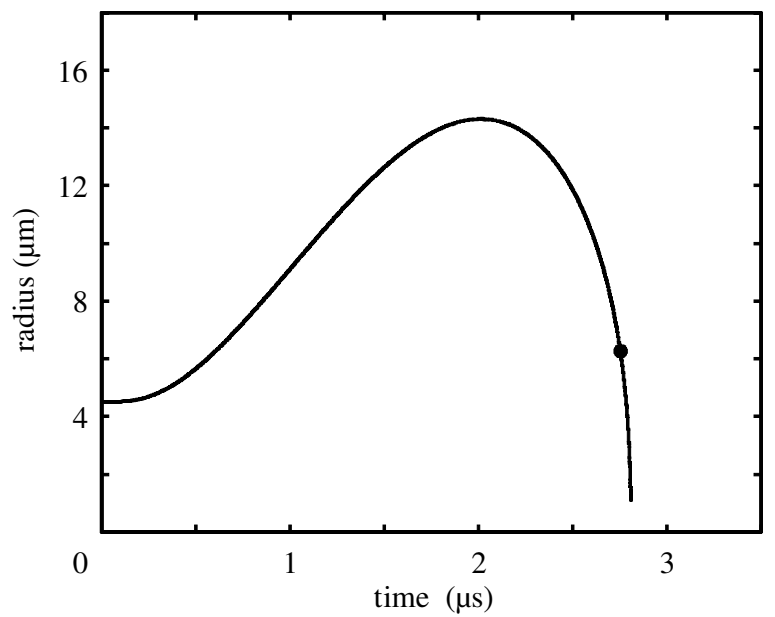

Figure 1. Radius of an argon bubble in liquid water versus time. We assume transient cavitation and stop the calculation when the bubble collapses to minimum radius. The 'dot' indicates when $\tau_{\text {diff }}=\tau_{\text {dyn }}$. The input parameters are $R_{0}=4.5 \mu \mathrm{m}, P_{\mathrm{A}}=2.0 \mathrm{~atm}, T_{0}=298 \mathrm{~K}, P_{0}=1 \mathrm{~atm}$, and $\omega=300 \mathrm{kHz}$.

This uniform isothermal behaviour continues into the collapse until the motions become rapid enough that the time-scale arguments of $\S \S 2 c$ and $2 d$ show that the bubble is collapsing adiabatically with constant mass. This time is indicated by the dot in figure 1 . To simplify matters we average the mass- and heat-diffusion timescales to create a single diffusive time-scale, $\tau_{\text {diff. }}$. Because the Prandtl number and Schmidt number usually differ little, this approximation is straightforward to justify. Therefore, when $\tau_{\text {diff }}=\tau_{\text {dyn }}$ we switch from an isothermal, variable-mass process to an adiabatic, constant-mass compression. In this part of the cycle we solve (2.1) and (2.2) for the radial dynamics, (2.4) for the number of moles of all the species, and (2.6) for the pressure. Equation (2.3) has a right-hand side of zero during this part of the cycle. We stop the calculation when the velocity of the collapse reaches zero on the assumption that the bubble breaks apart.

The behaviour just described is not general. For example, there are conditions where the expansion occurs adiabatically. One must always monitor the diffusive time-scales to be certain that the model is appropriate or adjust the model in a suitable way. For the conditions of interest in this work the description is valid. The model works well for our conditions primarily because there is very little time when the diffusive and dynamic time-scales are of equal magnitude. We note that it is trivial to modify the model to compute an entire acoustic cycle, if one wants to assume that the integrity of the bubble is maintained through the collapse. This model will not work well if $\tau_{\text {diff }} \approx \tau_{\text {dyn }}$ for much of the cycle.

\section{Comparison of the reduced and detailed models}

In this section we compare the conditions at the collapse predicted by the present, reduced model with the more detailed model presented in S\&S. Table 1 shows a summary of the parameters and conditions in the bubble at the point of minimum radius. 
Table 1. Comparison between the detailed computational results of Storey 8 Szeri (2000) and the present, reduced model

(From the detailed model we show the peak temperature at the centre, the mass percent of argon at the centre, and the number of $\mathrm{OH}$ molecules (scaled by the total number of molecules initially in the bubble). To obtain the total number of $\mathrm{OH}$ molecules, multiply the result in the table by $\left(P_{0}+2 \sigma / R_{0}\right)\left(\frac{4}{3} \pi R_{0}^{3}\right) / \bar{R} T_{0}$. For all cases in this table the dissolved gas is argon and $P_{0}=1 \mathrm{~atm}, T_{0}=298 \mathrm{~K}$, and $\sigma=72 \mathrm{mN} \mathrm{m}^{-1}$. The table is sectioned to show trends where only one parameter is varied, indicated by the extra horizontal space. The star denotes the base case that was analysed in detail in S\&S. This entry is repeated to show the trends more clearly.)

\begin{tabular}{|c|c|c|c|c|c|c|c|c|}
\hline \multicolumn{3}{|c|}{ parameter } & \multicolumn{3}{|c|}{ results of $S \& S$} & \multicolumn{3}{|c|}{ current results } \\
\hline $\begin{array}{c}R_{0} \\
(\mu \mathrm{m})\end{array}$ & $\begin{array}{c}P_{\mathrm{A}} \\
\text { (bar) }\end{array}$ & $\begin{array}{c}\omega \\
(\mathrm{kHz})\end{array}$ & $\begin{array}{c}T \\
(\mathrm{~K})\end{array}$ & $\begin{array}{c}\mathrm{OH} \\
(\times 100)\end{array}$ & $\begin{array}{c}\mathrm{Ar} \\
(\% \text { mass })\end{array}$ & $\begin{array}{c}T \\
(\mathrm{~K})\end{array}$ & $\begin{array}{c}\mathrm{OH} \\
(\times 100)\end{array}$ & $\begin{array}{c}\mathrm{Ar} \\
(\% \text { mass })\end{array}$ \\
\hline 4.5 & 1.10 & 26.5 & 4840 & 0.89 & 95 & 3520 & 0.33 & 96 \\
\hline 4.5 & 1.15 & 26.5 & 6380 & 2.97 & 91 & 4670 & 4.57 & 92 \\
\hline $4.5^{*}$ & 1.20 & 26.5 & 7030 & 5.29 & 86 & 5370 & 8.81 & 87 \\
\hline 4.5 & 1.25 & 26.5 & 6980 & 8.19 & 80 & 5600 & 12.2 & 81 \\
\hline 4.5 & 1.30 & 26.5 & 6870 & 12.2 & 72 & 5560 & 14.4 & 74 \\
\hline 2.0 & 1.20 & 26.5 & 6820 & 1.67 & 95 & 4326 & 2.16 & 96 \\
\hline 3.0 & 1.20 & 26.5 & 7560 & 4.31 & 89 & 5530 & 7.96 & 89 \\
\hline $4.5^{*}$ & 1.20 & 26.5 & 7030 & 5.29 & 86 & 5370 & 8.81 & 87 \\
\hline 6.5 & 1.20 & 26.5 & 6430 & 5.42 & 85 & 5060 & 8.24 & 87 \\
\hline 10.0 & 1.20 & 26.5 & 5640 & 4.93 & 85 & 4500 & 6.40 & 86 \\
\hline $4.5^{*}$ & 1.20 & 26.5 & 7030 & 5.29 & 86 & 5370 & 8.81 & 87 \\
\hline 4.5 & 1.20 & 38.3 & 6550 & 3.31 & 91 & 4830 & 5.39 & 92 \\
\hline 4.5 & 1.20 & 58.9 & 5712 & 1.88 & 94 & 4160 & 2.77 & 94 \\
\hline
\end{tabular}

Table 1 shows a comparison of the peak temperature, the amount of water trapped by the collapse, and the amount of $\mathrm{OH}$ produced. Because the total number of moles changes due to reactions, we use the mass fraction of non-condensible gas to compare the amount of water trapped by the collapse. In general, the reduced model produces reasonable results.

In actuality, the temperature and distribution of species are not uniform inside the bubble. Only a single value is computed in the reduced model, which makes comparing the two approaches awkward. For example, in table 1 we present the mass fraction of argon at the centre from S\&S. At the collapse the mass fraction of argon is lowest at the centre and highest at the wall. The average mass fraction of argon is therefore higher than the value at the centre. In fact, for the cases listed in table 1 the mass fraction at the centre and the average value always bracket the value determined by the reduced model. While a direct comparison is awkward, the reduced model is clearly producing reasonable results.

The most important thing to realize from table 1 is that the reduced model is capable of reproducing the trends in the data. For example, the first five entries show all parameters held constant except the acoustic forcing. Both methods predict that the temperature reaches a maximum, then decreases slightly with $P_{\mathrm{A}}$. Both methods also predict that the increased forcing will trap more water vapour in the bubble 
interior and produce more $\mathrm{OH}$. The trends with variation of ambient radius and frequency are captured as well. The fact that such trends are captured is important for parameter studies, as optimizing a sonochemical process with the reduced model should give very similar results to the more detailed model.

\section{Use of the reduced model in the study of sonochemistry}

In the previous section it was shown that the reduced model provides reasonable results for a single bubble. In this section we will demonstrate that the reduced model applied to a single bubble can account for some of the trends observed in sonochemistry data.

The difficulty in making direct use of the single-bubble model to predict sonochemistry data is in determining the input parameters to characterize the sonochemical bubble cloud, i.e. the mean radius of collapsing bubbles, the bubble number density, the driving pressure amplitude felt by the bubble, etc. These uncertainties make it difficult to determine if an effect one measures on changing an experimental parameter is due to changes in individual bubble dynamics or due to changes in the bubble population. Furthermore, it is not known how the chemical species produced by the bubble collapse are dispersed in the liquid. We simply assume that the species contained in the bubble at the end of the collapse will be completely dispersed in the liquid.

We emphasize that the purpose of this section is not to make quantitative predictions. Our purpose is to demonstrate the utility and the limitations of using a single-bubble model in sonochemistry. Single-bubble behaviour is commonly used to explain many of the trends in sonochemistry. In future work, this single-bubble model will become one component of a more complete model including population dynamics. These issues are discussed by Neppiras (1980) and Leighton (1995).

Despite all the uncertainties and unknowns, we will demonstrate that a single bubble is able to reproduce some trends and give insight into others. For all the cases we discuss in what follows, we assumed the input parameters: $R_{0}=4.5 \mu \mathrm{m}$, $P_{\mathrm{A}}=2.0 \mathrm{~atm}, T_{0}=298 \mathrm{~K}, P_{0}=1 \mathrm{~atm}$, and $\omega=300 \mathrm{kHz}$. The choice of $P_{\mathrm{A}}, T_{0}$ and $\omega$ are based on typical sonochemistry conditions, while the choice of $R_{0}$ is somewhat arbitrary. Variations of these input parameters were found to provide similar trends as the ones we present, though there are quantitative differences.

\section{(a) Helium-argon mixtures}

Mark et al. (1998) measured sonochemical yields of $\mathrm{OH}$ and $\mathrm{H}_{2} \mathrm{O}_{2}$ as the composition of a helium-argon gas mixture was varied. Our normalized predictions of the yields of $\mathrm{OH}$ and $\mathrm{H}_{2} \mathrm{O}_{2}$ as the fraction of helium is increased are shown in figure 2 . The solubility of the different species is taken into account with the results plotted versus fraction of helium in the liquid (which will not equal the fraction in the gas). The trend in this figure is similar to that measured by Mark et al. (1998) shown in the inset. Note that helium and argon have very different solubilities; hence a significant change in $R_{0}$ due to rectified diffusion is a possibility we have not taken into account. Such questions belong properly to a study of the evolution of bubble clouds, a subject outside the scope of the present work. The details of the shape of the curve are quite sensitive to the input parameters, but the general trend is very robust. 


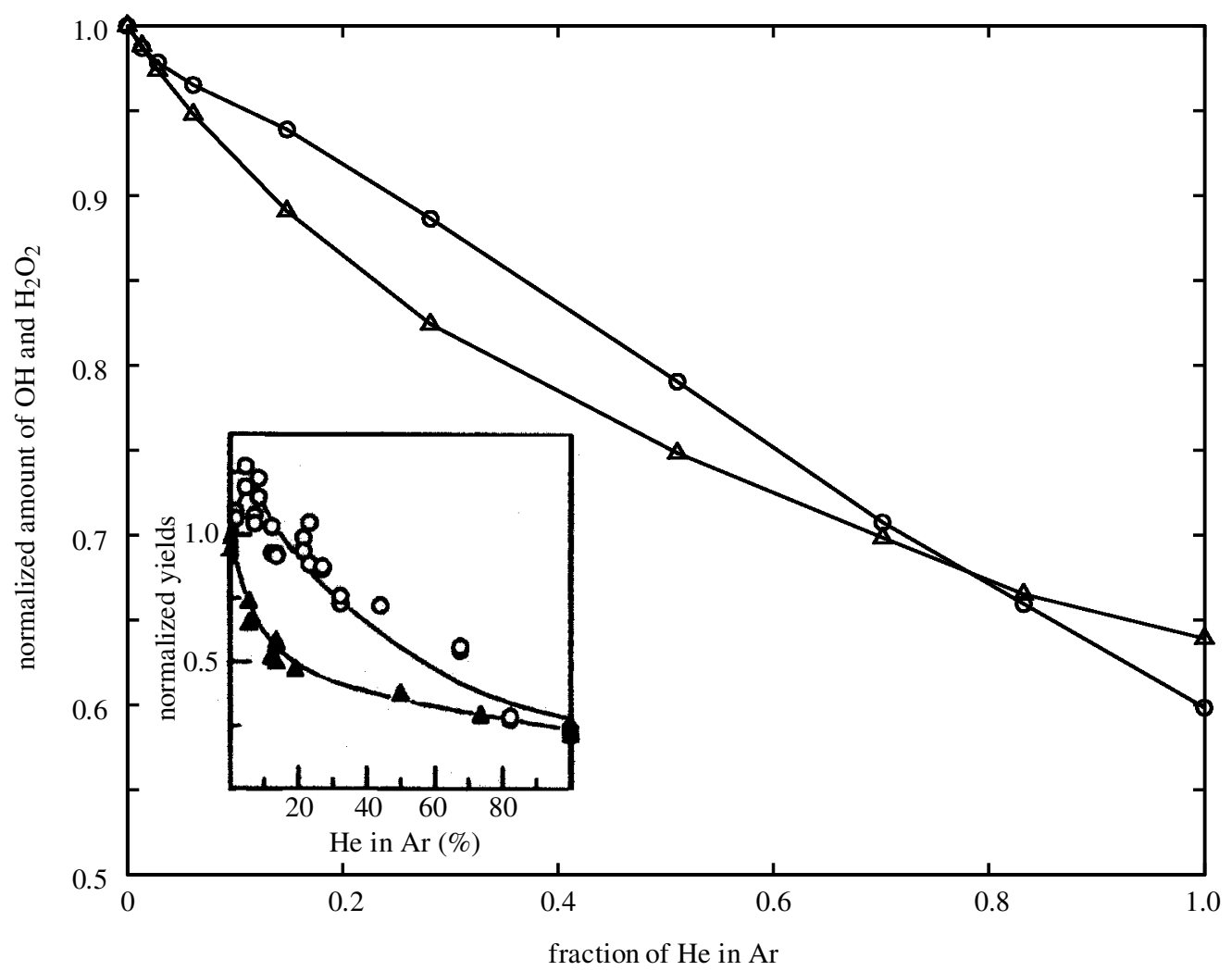

Figure 2. Normalized yields of $\mathrm{OH}$ and $\mathrm{H}_{2} \mathrm{O}_{2}$ as the amount of helium in argon is varied. Note that the results are plotted as helium fraction in argon dissolved in the liquid (different from helium fraction inside the bubble). The experimental data of normalized yields are shown in the inset (reprinted from Mark et al. (1998), with permission from Elsevier Science): the data are $\mathrm{H}_{2} \mathrm{O}_{2}$ (open circles) and $\mathrm{OH}$ (solid triangles) production.

Mark et al. (1998) speculated that the trend in decreasing $\mathrm{OH}$ and $\mathrm{H}_{2} \mathrm{O}_{2}$ yields was due to the increasing thermal conductivity with helium content. This view has also been put forth by many others (see Young 1989, p. 326). The speculation was that more heat loss leads to lower temperatures and therefore less chemical activity. The present model suggests an alternative interpretation. We find that as the helium content increases, the water vapour has increased mobility and can more easily diffuse out of the bubble interior. Therefore, as the helium content increases, the amount of trapped water decreases. The decrease in water content reduces the 'fuel' for $\mathrm{OH}$ and $\mathrm{H}_{2} \mathrm{O}_{2}$. Despite the increase in thermal conductivity we find that the temperature in the bubble increases (4400-4800 K) with helium content because there is less water to undergo reactions and absorb energy. Therefore, the decrease in the amount of water trapped in the bubble is the reason why $\mathrm{OH}$ and $\mathrm{H}_{2} \mathrm{O}_{2}$ yields decrease with increasing helium concentration.

The stark difference between our explanation based on vapour content and the explanation relying on thermal conductivity may be appreciated by examination of a calculation in which phase change is artificially prohibited. In this case, one finds that a pure argon bubble achieves a temperature of $9200 \mathrm{~K}$, compared with a pure 


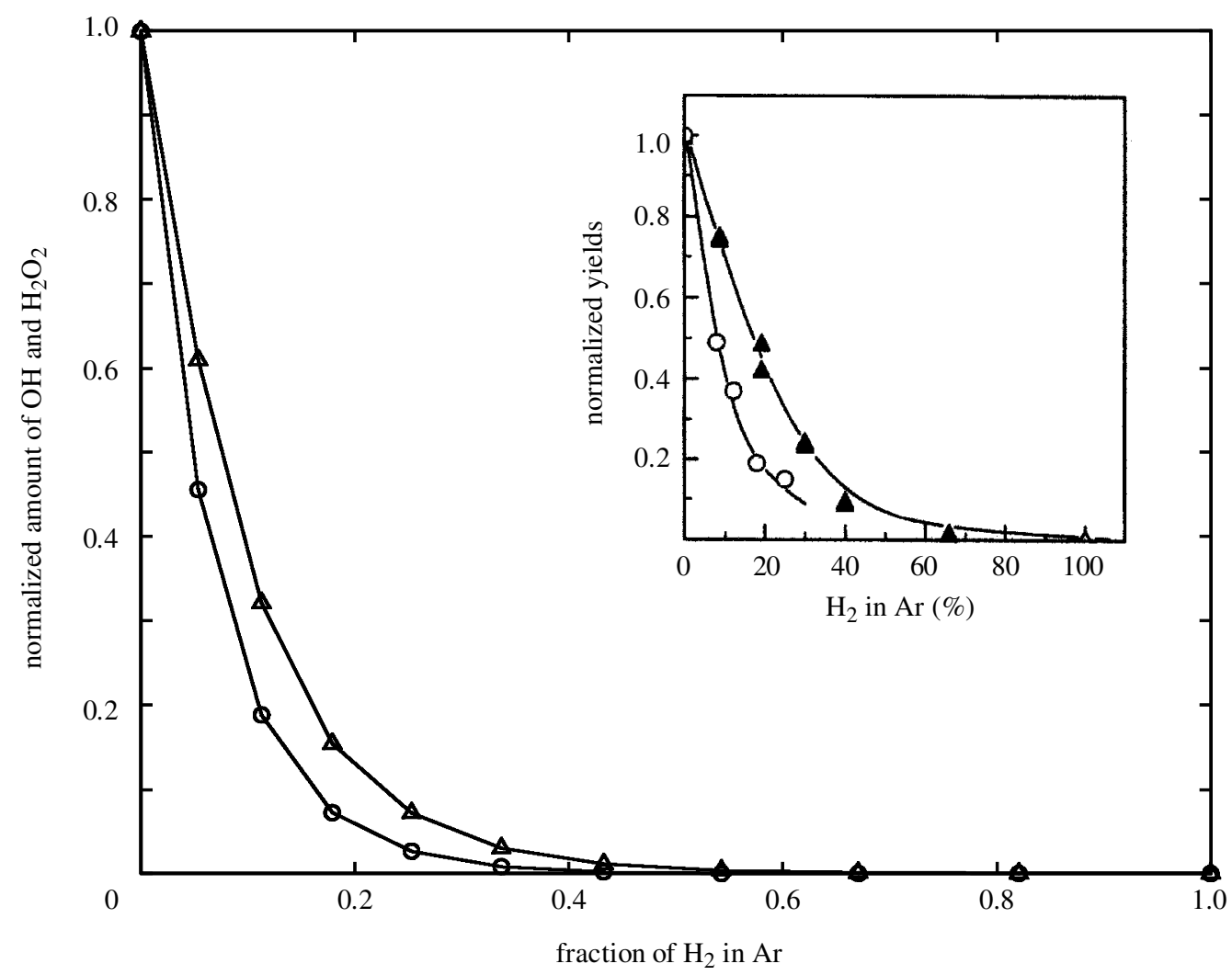

Figure 3. Normalized yields of $\mathrm{OH}$ and $\mathrm{H}_{2} \mathrm{O}_{2}$ as the amount of hydrogen in argon is varied. Note that the results are plotted as hydrogen fraction in argon dissolved in the liquid. The experimental data of normalized yields are shown in the inset (reprinted from Mark et al. (1998), with permission from Elsevier Science): the data are $\mathrm{H}_{2} \mathrm{O}_{2}$ (open circles) and $\mathrm{OH}$ (solid triangles) production.

helium bubble which achieves a temperature of $6600 \mathrm{~K}$. The lower temperature for the helium bubble in this instance is due to the higher thermal conductivity, which does indeed promote a greater loss of the heat of compression. We emphasize that this result is not physical, as the vapour dynamics are artificially suppressed.

When vapour is properly taken into account, the robust trend is for the quantity of water vapour trapped in a helium bubble to be much diminished compared with the quantity trapped in an argon bubble, with the consequence that the temperatures achieved are largely equal, notwithstanding the differences in thermal conductivity. The ramifications for chemistry are diminished production of $\mathrm{OH}$ and $\mathrm{H}_{2} \mathrm{O}_{2}$ in a helium bubble, all other things being equal. This example highlights the interdependence of heat and mass transfer. Heat- and mass-transfer effects must be considered together, not separately, when trying to understand the role of diffusive transport.

\section{(b) Hydrogen-argon mixtures}

Mark et al. (1998) also measured sonochemical yields as the composition of a hydrogen-argon mixture was varied. In figure 3 , we show the normalized sonochem- 
ical yields of $\mathrm{OH}$ and $\mathrm{H}_{2} \mathrm{O}_{2}$ that are predicted by the present model as one varies the $\mathrm{H}_{2}$ fraction in the liquid. The trend is very similar to that of the experimental data (inset). The sharp decline in the yields with increased hydrogen content is due to several factors:

(a) a chemical effect due to an increase in the amount of hydrogen,

(b) a chemical effect due to a decrease in water vapour content (which is due to the increased mass diffusivity),

(c) a decrease in the ratio of specific heats of the gas mixture, and

(d) an increase in thermal conductivity.

The chemical effects (a) and (b) are easiest to understand in terms of equilibrium chemistry. For a fixed temperature, pressure and water content, the chemical equilibrium amount of $\mathrm{OH}$ and $\mathrm{H}_{2} \mathrm{O}_{2}$ decreases significantly as the fraction of $\mathrm{H}_{2}$ increases (a). The same is true if we hold the fraction of $\mathrm{H}_{2}$ constant and decrease the fraction of $\mathrm{H}_{2} \mathrm{O}$ (b). Therefore, much of the decrease in the sonochemical yields is simply due to the change in the composition of the gas-vapour mixture that undergoes heating. We note that owing to the high density at collapse, the reactions occur very rapidly and are always near equilibrium. Therefore, interpreting the results in terms of chemical equilibrium is quite valid, and could form the basis of further simplifications.

Factors (c) and (d) lead to a decrease in the collapse temperature as hydrogen is added. The lower ratio of specific heats of hydrogen reduces the compression heating and the higher thermal conductivity removes more heat. Therefore, all four factors act together to decrease the amount of $\mathrm{OH}$ and $\mathrm{H}_{2} \mathrm{O}_{2}$ as the fraction of hydrogen is increased. This general trend is very robust with changes in the input parameters.

\section{(c) Oxygen-argon mixture}

Hart \& Henglein (1985) measured sonochemical yields of $\mathrm{H}_{2} \mathrm{O}_{2}$ as the composition of an oxygen-argon mixture was varied. The normalized sonochemical yields we compute as a function of $\mathrm{O}_{2}$ content are shown in figure 4. Again, we obtain reasonable agreement between the single-bubble model and the experimental data (inset). We show the predicted results for two different pressure amplitudes, with the $P_{\mathrm{A}}=3.0$ curve looking very similar to the experimental results. The shape of the curve and the location of the maximum are dependent on the input parameters, but the general trend is very robust.

The maximum in the data is a consequence of competing effects. As the oxygen is introduced, equilibrium chemistry dictates that there should be a monotonic increase in $\mathrm{H}_{2} \mathrm{O}_{2}$ if all parameters are held constant (i.e. temperature, pressure and water content). On the other hand, as the amount of $\mathrm{O}_{2}$ increases, the gas mixture has a lower specific heat ratio, reducing the peak temperature. These offsetting effects lead to the maximum in the $\mathrm{H}_{2} \mathrm{O}_{2}$ yields. Heat and mass diffusion play a minor role because the diffusive coefficients for argon and oxygen are similar. 


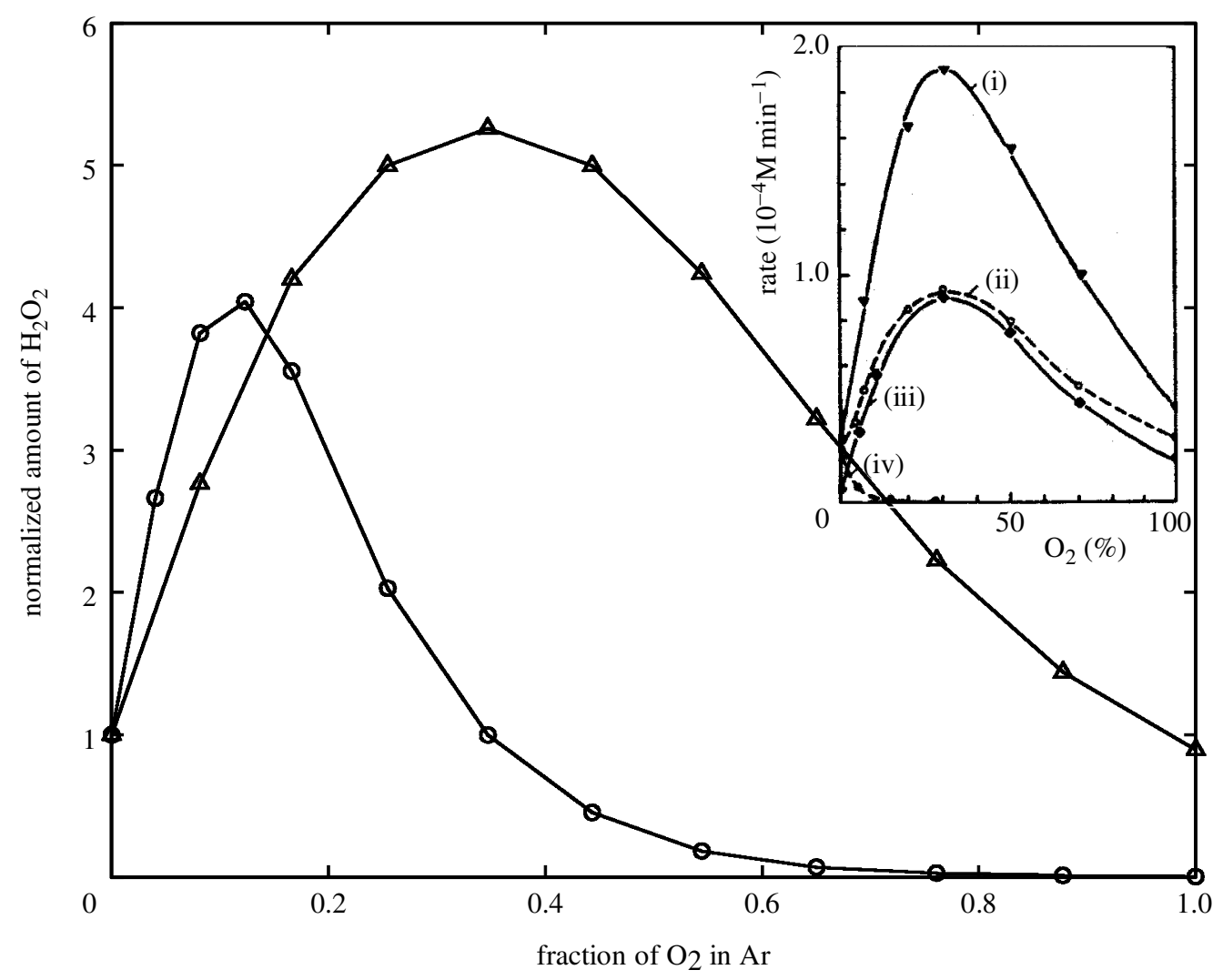

Figure 4. Normalized yields of $\mathrm{H}_{2} \mathrm{O}_{2}$ as the amount of oxygen in argon is varied. Curves are shown for two different pressure amplitudes $\left(P_{\mathrm{A}}=2.0\right.$, open circles; $P_{\mathrm{A}}=3.0$, open triangles $)$. Note that the results are plotted as oxygen fraction in argon dissolved in the liquid. The experimental data are shown in the inset (reprinted from Hart \& Henglein (1985), with permission from the American Chemical Society): the data fitted with the dashed curve are $\mathrm{H}_{2} \mathrm{O}_{2}$ yields. The solid curves in the inset are not relevant to the current study. The labels in the inset refer to (i) $\mathrm{I}_{2}$ in an aqueous $0.1 \mathrm{M}$ potassium iodide and $0.0005 \mathrm{M}$ ammonium molybdate solution; (ii) $\mathrm{H}_{2} \mathrm{O}_{2}$ in water; (iii) $\mathrm{I}_{2}$ in a $0.1 \mathrm{M}$ potassium iodide solution; (iv) $\mathrm{H}_{2}$ in water.

\section{(d) Acoustic power}

Mark et al. (1998) measured sonochemical yields of $\mathrm{OH}$ as the acoustic power input is increased. The predicted $\mathrm{OH}$ yields as the pressure amplitude is varied between 1 and $10 \mathrm{~atm}$ are shown in figure 5 . The model predicts an initial increase in yields which then reach a plateau; a trend qualitatively similar to the experimental data (inset). The experimental data were taken at various frequencies, but the general trend is very robust. While a quantitative comparison is impossible owing to the uncertainties in the model parameters, the single-bubble model is able to predict the proper trend. The plateau in the yields is due to offsetting effects. The higher acoustic forcing causes the bubbles to collapse more violently, but the more violent collapse also traps more vapour in the bubble. This trend would not be captured if the diffusion of vapour in the interior had not been taken into account. 


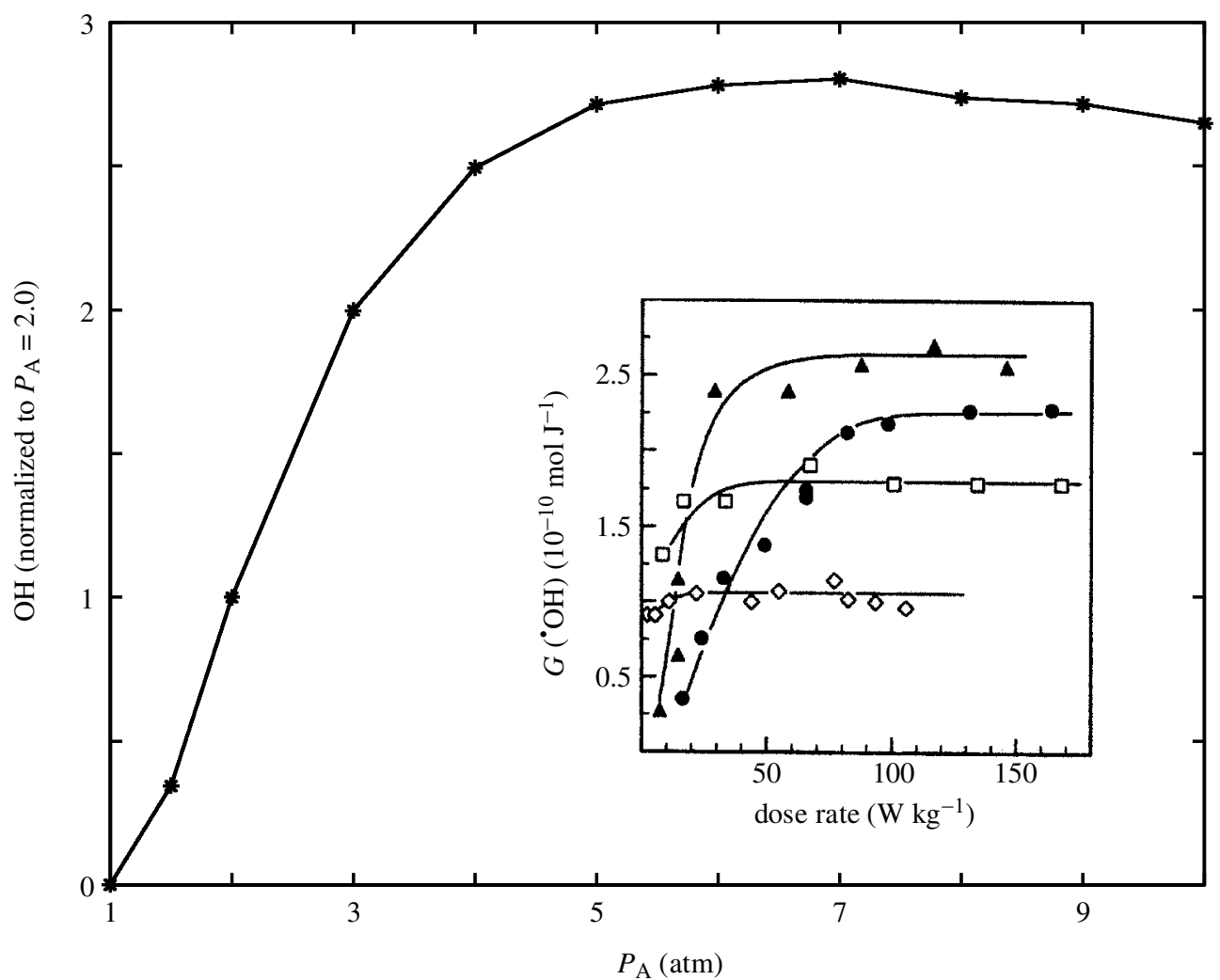

Figure 5. Yields of $\mathrm{OH}$, normalized to $P_{\mathrm{A}}=2.0$, as the pressure amplitude is varied. Experimental data of $\mathrm{OH}$ sonochemical yields for various frequencies as power is varied are shown in the inset (reprinted from Mark et al. (1998), with permission from Elsevier Science). The important experimental result is that for all frequencies shown, the chemical yields reach a plateau as the power is increased.

\section{(e) Liquid temperature}

Finally, Mark et al. (1998) measured sonochemical yields of argon dissolved in water as the liquid temperature is varied. The predicted normalized $\mathrm{OH}$ yields as the liquid temperature is varied are shown in figure 6 . The experimental result shows a monotonic decrease in $\mathrm{OH}$ and $\mathrm{H}_{2} \mathrm{O}_{2}$ as the liquid temperature increases. As the liquid temperature increases, the vapour pressure increases and consequently more vapour is trapped by the collapse. The increased vapour content causes the temperature within the collapsing bubbles to decrease monotonically as the liquid temperature increases. At low liquid temperatures, the collapse temperature is high because the bubble is nearly pure argon, but there is not enough water vapour present to produce significant yields. At high liquid temperatures, there is too much vapour to yield a significant rise in temperature. These competing effects lead to the maximum in the predicted single-bubble sonochemical yields.

The experimental trend with temperature (which is also observed in sonoluminescence) is often explained in a simple way: i.e. the increase in vapour content with temperature results in less compression heating, which results in fewer reactions. 


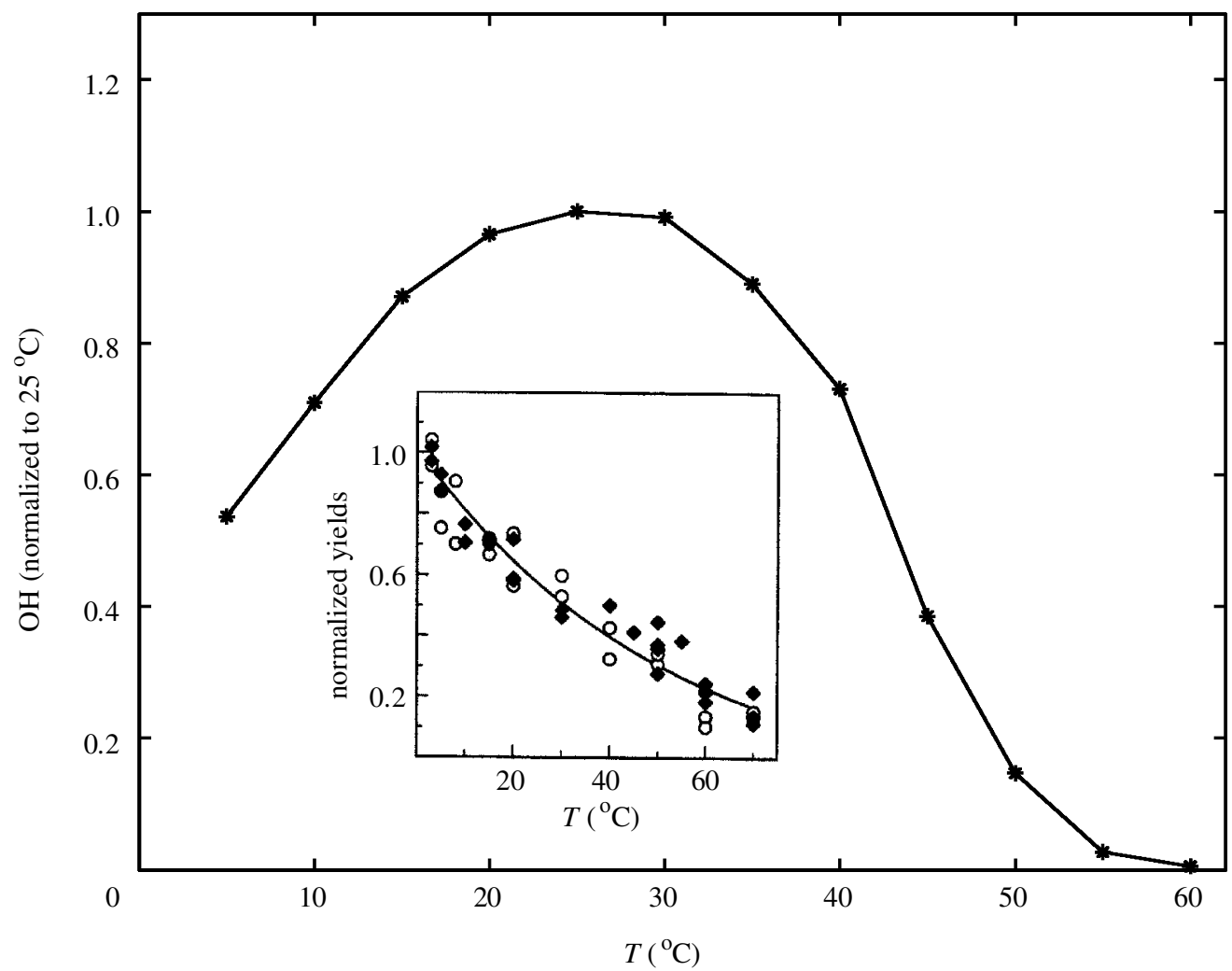

Figure 6. Yields of $\mathrm{OH}$, normalized to $298 \mathrm{~K}$, as the liquid temperature is varied. Experimental data of sonochemical yields versus temperature are shown in the inset (reprinted from Mark et al. (1998), with permission from Elsevier Science).

While this explanation does explain the peak bubble temperature, the interpretation of the sonochemical yields is incomplete, as shown by the inability of the model to predict the experimental trend. The discrepancy between the experimental and predicted trend could be due to changes in ambient bubble radius, changes in the bubble population density, changes in the uptake of $\mathrm{OH}$ and $\mathrm{H}_{2} \mathrm{O}_{2}$ by the liquid, or other differences, which make a strict analogy between single- and multi-bubble behaviour inappropriate.

\section{Conclusions}

We have developed a reduced model that captures the physics of violent bubble collapses relevant to sonochemistry. The model only requires a minor extension and modification to the traditional Rayleigh-Plesset equation, which is solved easily numerically with widely available methods. The model also involves a coupling with chemical kinetics, which can be included using widely available software. The model is a simple and practical tool for understanding the sonochemical yields of single bubbles.

We have shown that this model is reasonably accurate in predicting the general trends of single-bubble behaviour by comparison with our previous work (S\&S) 
involving much more detailed methods. The reduced model provides reasonable predictions for sonochemical yields and peak temperatures. Most importantly, the model is useful in predicting the amount of water present during the collapse, which is crucial for determination of the sonochemical yields. The importance of vapour has been widely ignored in the literature.

The reduced model was employed in the study of multi-bubble sonochemistry data. The model works well in predicting trends with variations in the operating gas, yielding the same general trends as the experimental data for monatomic gas mixtures and monatomic-diatomic gas mixtures. Furthermore, the model is able to shed light on the influence of variations in acoustic power. However, the single-bubble model is not able to predict the experimental data for variations in liquid temperature. This issue highlights the shortcomings of applying a single-bubble model to complex multi-bubble situations.

There are many unknowns in multi-bubble sonochemistry, such as the bubble size distribution, bubble population density, bubble-bubble interactions, and the mechanism by which chemical species produced in the interior are dispersed in the liquid. In theory, this single-bubble model could be part of a more complete theory if many of these unknowns could be resolved. The model is simple enough that it could be applied to an evolving size and number distribution of bubbles in a cloud. Further research is needed in the characterization of multi-bubble fields before accurate quantitative sonochemistry models can be expected.

This work was supported by the US National Science Foundation and Lawrence Livermore National Laboratory.

\section{References}

Carey, V. P. 1992 Liquid-vapor phase change phenomena. Taylor \& Francis.

Colussi, A. J. \& Hoffmann, M. R. 1999 Vapor supersaturation in collapsing bubbles. Relevance to the mechanisms of sonochemistry and sonoluminescence. J. Phys. Chem. A 103, 1133611339 .

Colussi, A. J., Weavers, L. K. \& Hoffmann, M. R. 1998 Chemical bubble dynamics and quantitative sonochemistry. J. Phys. Chem. A 102, 6927-6934.

Fujikawa, S. \& Akamatsu, T. 1980 Effects of the non-equilibrium condensation of vapour on the pressure wave produced by the collapse of a bubble in a liquid. J. Fluid Mech. 97, 481-512.

Gardiner, W. C. 1984 Combustion chemistry. Springer.

Gong, C. \& Hart, D. P. 1998 Ultrasound induced cavitation and sonochemical yields. J. Acoust. Soc. Am. 104, 2675-2682.

Hart, E. J. \& Henglein, A. 1985 Free radical and free atom reactions in the sonolysis of aqueous iodide and formate solutions. J. Phys. Chem. $894342-4347$.

Kamath, V., Prosperetti, A. \& Egolfopoulos, F. N. 1993 A theoretical study of sonoluminescence. J. Acoust. Soc. Am. 94, 248-260.

Kee, R. J., Rupley, F. M. \& Miller, J. A. 1994 CHEMKIN: a FORTRAN chemical kinetics package for the analysis of gas-phase chemical kinetics. Sandia National Laboratories.

Leighton, T. G. 1994 The acoustic bubble. Academic.

Leighton, T. G. 1995 Bubble population phenomena in acoustic cavitation. Ultrason. Sonochem. 2, S123-S136.

Maas, U. \& Warnatz, J. 1988 Ignition processes in hydrogen-oxygen mixtures. Combust. Flame 74, 53-69. 
Mark, G., Tauber, A., Laupert, R., Schechmann, H.-P., Schulz, D., Mues, A. \& von Sonntag, C. 1998 OH-radical formation by ultrasound in aqueous solution. Part II. Terephthalate and Fricke dosimetry and the influence of various conditions on the sonolytic yield. Ultrason. Sonochem. 5, 41-52.

Mason, T. J. 1999 Sonochemistry: current uses and future prospects in the chemical and processing industries. Phil. Trans. R. Soc. Lond. A 357, 355-369.

Moss, W. C., Young, D. A., Harte, J. A., Levatin, J. L., Rozsnyai, B. F., Zimmerman, G. B. \& Zimmerman, I. H. 1999 Computed optical emissions from a sonoluminescing bubble. Phys. Rev. E 59, 2986-2992.

Moss, W. C., Levatin, J. L. \& Szeri, A. J. 2000 A new damping mechanism in strongly collapsing bubbles. Proc. R. Soc. Lond. A 456, 2983-2994.

Neppiras, E. A. 1980 Acoustic cavitation. Phys. Rep. 61, 159-251.

Neppiras, E. A. \& Noltingk, B. E. 1951 Cavitation produced by ultrasonics: theoretical conditions for the onset of cavitation. Proc. Phys. Soc. B 64, 1032-1038.

Nigmatulin, R. I., Khabeev, N. S. \& Nagiev, F. B. 1981 Dynamics, heat and mass transfer of vapour-gas bubbles in a liquid. Int. J. Heat Mass Transfer 1981, 1033-1043.

Noltingk, B. E. \& Neppiras, E. A. 1950 Cavitation produced by ultrasonics. Proc. Phys. Soc. B 63, 674-685.

Plesset, M. S. \& Hsieh, D. Y. 1960 Theory of gas bubble dynamics in oscillating pressure fields. Phys. Fluids 3, 882-892.

Plesset, M. S. \& Zwick, S. A. 1954 The growth of vapor bubbles in superheated liquids. J. Appl. Phys. 25, 493-500.

Prosperetti, A. \& Lezzi, A. 1986 Bubble dynamics in a compressible liquid. Part 1. First-order theory. J. Fluid Mech. 168, 457-477.

Prosperetti, A., Crum, L. A. \& Commander, K. W. 1988 Nonlinear bubble dynamics. J. Acoust. Soc. Am. 83, 502-514.

Sochard, S., Wilhelm, A. M. \& Delmas, H. 1997 Modeling of free radicals production in a collapsing gas-vapour bubble. Ultrason. Sonochem. 4, 77-84.

Storey, B. D. \& Szeri, A. J. 1999 Mixture segregation within sonoluminescence bubbles. J. Fluid Mech. 396, 203-221.

Storey, B. D. \& Szeri, A. J. 2000 Water vapour, sonoluminescence, and sonochemistry. Proc. $R$. Soc. Lond. A 456, 1685-1709.

Suslick, K. S., Didenko, Y., Fang, M. F., Hyeon, T., Kolbeck, K. J., McNamara, W. B., Mdleleni, M. M. \& Wong, M. 1999 Acoustic cavitation and its chemical consequences. Phil. Trans. R. Soc. Lond. A 357, 335-353.

Thompson, L. H. \& Doraiswamy, L. K. 1999 Sonochemistry: science and engineering. Ind. Engng Chem. Res. 38, 1215-1249.

von Sonntag, C., Mark, G., Tauber, A. \& Schuchmann, H.-P. 1999 OH radical formation and dosimetry in the sonolysis of aqueous solutions. Adv. Sonochem. 5, 109-145.

Vuong, V. Q. \& Szeri, A. J. 1996 Sonoluminescence and diffusive transport. Phys. Fluids 8, 2354-2364.

Yasui, K. 1997a Alternative model of single-bubble sonoluminescence. Phys. Rev. E 56, 67506760.

Yasui, K. $1997 b$ Chemical reactions in a sonoluminescing bubble. J. Phys. Soc. Jpn 66, 29112920.

Young, F. R. 1989 Cavitation. McGraw-Hill. 